\title{
On the Probability of Dizygotic Twins Being Concordant for Two Alleles at Multiple Polymorphic Loci
}

\author{
Dale R. Nyholt \\ Queensland Institute of Medical Research, Brisbane, Australia
}

\begin{abstract}
ccurate determination of same-sex twin zygosity is important for medical, scientific and personal reasons. Determination may be based upon questionnaire data, blood group, enzyme isoforms and fetal membrane examination, but assignment of zygosity must ultimately be confirmed by genotypic data. Here methods are reviewed for calculating average probabilities of correctly concluding a twin pair is monozygotic, given they share the same genotypes across all loci for commonly utilized multiplex short tandem repeat (STR) kits. Numerous tools enabling convenient and accurate zygosity probability calculation may be accessed via the ZygProb homepage at http://genepi.qimr.edu.au/general/daleN/ZygProb/
\end{abstract}

Accurate determination of twin zygosity is important for medical, scientific and personal reasons (Derom et al., 2001). Typically, questionnaire-based assessment of same-sex twin pair zygosity is relied upon, using questions about physical similarity and confusion in childhood. Example questions utilized in our laboratory include: Do you have the same eye colour? Do you have similar height, weight, and natural hair colour and texture? Were you usually mistaken for one another by nonfamily members as children? If the answer to these questions is 'yes', the pair is almost certainly monozygotic (MZ).

While most such methods combine information from both twins from a pair, answers from a single twin were found to yield a misclassification rate below $5 \%$ (Magnus et al., 1983). However, an approach utilizing latent class analysis was recently introduced which further improved the accuracy of questionnairebased zygosity assessment (Heath et al., 2003). Nonetheless, while MZ twins usually have closely similar phenotypes they are seldom absolutely 'identical' - indeed some MZ twin pairs show quite marked phenotypic discordances. Moreover, it is preferable to determine zygosity at birth; examination of fetal membranes by properly trained personnel enables diagnosis of only two thirds of MZ twin pairs since one third are dichorionic (Derom et al., 2001). Consequently, assignment of zygosity must ultimately be confirmed by genotypic data, for example by utilizing the
AMPFlSTR Profiler Plus ${ }^{\text {TM }}$ PCR Amplification Kit (Applied Biosystems), widely used in forensic and paternity applications.

The desire to gain more information from a sample, coupled with the need to limit consumption of a DNA sample where its availability may be limited, has led to the coamplification and typing of multiple short tandem repeat (STR) systems. Kits from commercial sources, namely Promega and Applied Biosystems, are preferred due to their ease of use. Commonly utilized multiplex STR kits are listed in Table 1.

Given the multiplex kits listed in Table 1 differ in both number and site of STR loci, it is important to examine their differences. More specifically, we are interested in their respective average probabilities of correctly concluding a twin pair is $\mathrm{MZ}$ given they share the same genotypes across all loci.

Following the notation of $\mathrm{Li}$ (1996), Mendel's law of segregation dictates parents with genotypes $(b, c)$ and $(d, e)$ can produce four types of children $(b, d),(b, e),(c, d)$ and $(c, e)$ with equal probabilities. The $4 \times 4$ array

Table 1

Commonly Utilized Multiplex STR Kits

\begin{tabular}{llc}
\hline Manufacturer & Kit name (abbreviation) & Number of STRs \\
\hline Promega & PowerPlex 1.1 (PPlex1.1) & 8 \\
& PowerPlex 2.1 (PPlex2.1) & 9 \\
& PowerPlex 16 (PPlex16) & 15 \\
Applied Biosystems & AMPFISTR COfiler (COfiler) & 6 \\
& AMPFISTR Profiler (Profiler) & 9 \\
& AMPFISTR Profiler Plus (ProfilerP) & 9 \\
& AMPFISTR SGM Plus (SGMPlus) & 10 \\
& AMPFISTR SEfiler (SEfiler) & 11 \\
& AMPFISTR Identifier (Identifier) & 15 \\
\hline
\end{tabular}

Received 7 December, 2005; accepted 14 December, 2005.

Address for correspondence: Dale R. Nyholt, Queensland Institute of Medical Research, PO Royal Brisbane Hospital, Queensland 4029, Australia.E-mail:Dale.Nyholt@qimr.edu.au 


\begin{tabular}{|c|c|c|c|c|c|}
\hline \multicolumn{2}{|l|}{ parents } & \multicolumn{4}{|c|}{$(b, c) \times(d, e)$} \\
\hline children & & $(b, d)$ & $(b, e)$ & $(c, d)$ & $(c, e)$ \\
\hline \multirow[t]{4}{*}{ sib-pairs } & $(b, d)$ & 2 & 1 & 1 & 0 \\
\hline & $(b, e)$ & 1 & 2 & 0 & 1 \\
\hline & $(c, d)$ & 1 & 0 & 2 & 1 \\
\hline & $(c, e)$ & 0 & 1 & 1 & 2 \\
\hline
\end{tabular}

\section{Figure 1}

The three types of DZ twin pairs from a cross in which all parental alleles can be uniquely identified.

Note: Genotypes are represented by letters within brackets. Numbers represent the number of alleles shared identical by descent (IBD).

(Figure 1) shows the 16 possible dizygotic (DZ) twin pairs (/sib-pairs) arising from a fully informative mating.

Based on the number of alleles shared identical by descent (IBD) between the 16 possible pairs, there are three types of DZ pairs: (1) those sharing no alleles $\left(z_{0}\right)$, with frequency $4 / 16=1 / 4 ;(2)$ those sharing one allele $\left(z_{1}\right)$, with frequency $8 / 16=1 / 2$; and $(3)$ those sharing two alleles $\left(z_{2}\right)$, with frequency $4 / 16=1 / 4$. Therefore, the probability of a genotype match between two DZ twins, denoted $M(\mathrm{DZ})$, is as follows:

$$
M(\mathrm{DZ})=1 / 4 \operatorname{Prob}\left(z_{0}\right)+1 / 2 \operatorname{Prob}\left(z_{1}\right)+1 / 4 \operatorname{Prob}\left(z_{2}\right)
$$

When the two DZ twins have no alleles identical by descent $\left(z_{0}\right)$, they are like two unrelated individuals. Assuming a population in Hardy-Weinberg equilibrium, the probability of a genotype match between two random individuals is the sum of the frequencies of all pairs of identical genotypes. For simplicity, we first consider a locus with two alleles: allele $A_{i}$ and allele $A_{i}$, with frequencies $p_{i}$ and $p_{i}$, and genotypes $A_{i} A_{i}$ and $A_{i} A_{i}$, with frequencies $\left(A_{i} A_{i}\right)$ and $\left(A_{i} A_{j}\right)$. The frequency of two random individuals both being $A_{i} A_{i}$ is $\left(p_{i}\right)^{2} \times\left(p_{i}\right)^{2}=\left(p_{i}\right)^{4}$ and the frequency of two random individuals both being $A_{i} A_{j}$ is $\left(2 p_{i} p_{j}\right) \times\left(2 p_{i} p_{j}\right)=\left(2 p_{i} p_{j}\right)^{2}$. Therefore, the random match probability for two random individuals,

$$
\begin{aligned}
M_{0} & =\sum_{i=1}^{n}\left(A_{i} A_{i}\right)^{2}+\sum_{i=1}^{n} \sum_{j=i+1}^{n}\left(A_{i} A_{j}\right)^{2} \\
& =\sum_{i=1}^{n}\left(p_{i}\right)^{4}+\sum_{i=1}^{n} \sum_{j=i+1}^{n}\left(2 p_{i} p_{j}\right)^{2}
\end{aligned}
$$

For loci with more than two alleles $(n), i$ and $j$ take all values from 1 to $n$.

When two DZ twins have one allele identical by descent $\left(z_{1}\right)$, they are like a parent-child pair where the probability of a genotype match between a parent and child is the sum of the homozygous probabilities. The frequency of a random individual being $A_{i}$ is $\left(p_{i}\right)$ the frequency of both a parent and child both being $A_{i}$ is $\left(p_{i}\right)^{2}$. Therefore, the random match probability for a parent and child,

$$
a_{2}=\sum_{i=1}^{n}\left(p_{i}\right)^{2}
$$

When two DZ twins have both alleles identical by descent $\left(z_{2}\right)$, they are like MZ twins with a match probability of 1 . Therefore, the probability of a genotype match between two DZ twins, $M(\mathrm{DZ})$, is as follows:

$$
\begin{aligned}
M(\mathrm{DZ}) & =1 / 4 \operatorname{Prob}\left(z_{0}\right)+1 / 2 \operatorname{Prob}\left(z_{1}\right)+1 / 4 \operatorname{Prob}\left(z_{2}\right) \\
& =1 / 4 M_{0}+1 / 2 a_{2}+1 / 4 \\
& =1 / 4\left(\sum_{i=1}^{n}\left(p_{i}\right)^{4}+\sum_{i=1}^{n} \sum_{j=i+1}^{n}\left(2 p_{i} p_{j}\right)^{2}\right)+1 / 2 \sum_{i=1}^{n}\left(p_{i}\right)^{2}+1 / 4
\end{aligned}
$$

Using Australian Caucasian allele frequency data (Bagdonavicius et al., 2002) and the above exact $M(\mathrm{DZ})$ equation ( $\mathrm{Li}, 1996)$, the author calculated the average probability of a DZ twin pair sharing both alleles at all markers and the resulting probability of correct zygosity assignment for the commonly used multiplex STR kits COfiler, PPlex1.1, Profiler and ProfilerP for which allele frequency data were available (Table 2). That is, when the loci are unlinked, the allele sharing at each locus is independent and one simply multiplies together all individual marker $M(\mathrm{DZ})$ probabilities.

In addition to the exact equation presented above, Presciuttini and colleagues (Presciuttini et al., 2002) showed that the probabilities $\left(z_{i}\right)$ depend on locus heterozygosity $(H)$, and are scarcely affected by variation of the distribution of allele frequencies. This allowed them to obtain empirical curves relating $z_{i} \mathrm{~s}$ to $H$ for a series of common relationships, so that the likelihood ratio of a

\begin{tabular}{|c|c|c|c|c|}
\hline \multirow{2}{*}{ STR locus } & \multicolumn{4}{|c|}{ Common multiplex STR kits } \\
\hline & PPlex1.1 & COfiler & Profiler & ProfilerP \\
\hline TPOX & .486 & .486 & .486 & \\
\hline D5S818 & .431 & & .431 & .431 \\
\hline CSF1PO & .422 & .422 & .422 & \\
\hline D16S539 & .379 & .379 & & \\
\hline D13S317 & .372 & & .372 & .372 \\
\hline TH01 & .383 & .383 & .383 & \\
\hline D3S1358 & & .374 & .374 & .374 \\
\hline VWA & .362 & & .362 & .362 \\
\hline D8S1179 & & & & .357 \\
\hline D7S820 & .360 & .360 & .360 & .360 \\
\hline D21S11 & & & & .337 \\
\hline FGA & & & .326 & .326 \\
\hline D18S51 & & & & .319 \\
\hline Number of STRs & 8 & 6 & 9 & 9 \\
\hline Overall M(DZ) & $6.21 \mathrm{E}-04$ & 4.01E-03 & $2.00 \mathrm{E}-04$ & $9.79 \mathrm{E}-05$ \\
\hline $0 \mathrm{dds}_{\mathrm{Mz}: \mathrm{Dz}}$ & 1611 & 250 & 4993 & 10219 \\
\hline MZ Certainty ${ }_{\text {avg }}(\%)$ & 99.93792 & 99.59927 & 99.97997 & 99.99021 \\
\hline
\end{tabular}
pair of relationships between any two individuals, given their genotypes at a locus, is a function of a single parameter, $H$. Plotting the sharing probabilities for 19 STR

\section{Table 2}

Exact Probability of an Australian Caucasian DZ Pair Sharing the Same Two Alleles

Note: Utilizing allele frequency data from Bagdonavicius et al. (2002) and applying the exact M(DZ) formula of Li (1996). 
Table 3

Approximate Probability of an Australian Caucasian DZ Pair Sharing the Same Two Alleles

\begin{tabular}{|c|c|c|c|c|}
\hline \multirow[t]{2}{*}{ STR locus } & \multicolumn{4}{|c|}{ Common multiplex STR kits } \\
\hline & PPlex1.1 & COfiler & Profiler & ProfilerP \\
\hline TPOX & .489 & .489 & .489 & \\
\hline D5S818 & .431 & & .431 & .431 \\
\hline CSF1PO & .419 & .419 & .419 & \\
\hline D16S539 & .377 & .377 & & \\
\hline D13S317 & .372 & & .372 & .372 \\
\hline TH01 & .381 & .381 & .381 & \\
\hline D3S1358 & & .374 & .374 & .374 \\
\hline VWA & .362 & & .362 & .362 \\
\hline D8S1179 & & & & .357 \\
\hline D7S820 & .360 & .360 & .360 & .360 \\
\hline D21S11 & & & & .337 \\
\hline FGA & & & .326 & .326 \\
\hline D18S51 & & & & .319 \\
\hline Number of STRs & 8 & 6 & 9 & 9 \\
\hline Overall $M(D Z)_{\text {approx }}$ & 6.19E-04 & $3.96 \mathrm{E}-03$ & 1.99E-04 & $9.75 \mathrm{E}-05$ \\
\hline $\mathrm{Odds}_{\mathrm{MZ}: \mathrm{DZ}}$ & 1618 & 252 & 5036 & 10,252 \\
\hline MZ Certainty avg $_{(\%)}$ & 99.93820 & 99.60378 & 99.98014 & 99.99025 \\
\hline
\end{tabular}

Note: Utilizing expected heterozygosity $(\mathrm{H})$ values reported by Bagdonavicius et al. (2002) and applying the M(DZ) ${ }_{\text {approx }}$ formula of Presciuttini et al. (2002). loci relating to $H$ produced the following equation for a third order polynomial curve for the probability of a genotype match between two DZ twins, $M(\mathrm{DZ})_{\text {approx }}$ :

$$
\begin{aligned}
M(\mathrm{DZ})_{\mathrm{a}}= & 0.7753+0.0358 \times H-1.1771 \times H^{2} \\
& +0.6181 \times H^{3}
\end{aligned}
$$

Table 3 contains approximate average probability of a DZ twin pair sharing both alleles at all markers and resulting probability of correct zygosity assignment, for the commonly used multiplex STR kits COfiler, PPlex1.1, Profiler and ProfilerP utilizing the expected heterozygosity $(H)$ values reported by Bagdonavicius et al., (2002) and applying the $M(\mathrm{DZ})_{\text {approx }}$ formula of Presciuttini et al., (2002).

Upon comparing Table 2 and Table 3, it is clear the formula of Presciuttini et al. (2002) approximates the exact probabilities well. Furthermore, they report, because heterozygosity is a composite parameter it is inherently less variable among populations than individual allele frequencies. Hence heterozygosity is sufficiently homogeneous, at least among Caucasian populations, as to justify the adoption of a single common mean value, apart from special cases of historically isolated groups. Consequently researchers may simply utilize the marker $M(\mathrm{DZ})$ values reported by Presciuttini et al. (2002; and repeated in Table 4 below)

Table 4

\begin{tabular}{|c|c|c|c|c|c|c|c|c|c|}
\hline \multirow[t]{2}{*}{ STR locus } & \multicolumn{9}{|c|}{ Commonly utilized multiplex STR kits } \\
\hline & PPlex1.1 & PPlex2.1 & PPlex16 & COfiler & Profiler & ProfilerP & SGMPlus & SEfiler & Identifier \\
\hline TPOX & .483 & .483 & .483 & .483 & .483 & & & & .483 \\
\hline D5S818 & .422 & & .422 & & .422 & .422 & & & .422 \\
\hline CSF1PO & .414 & & .414 & .414 & .414 & & & & .414 \\
\hline D16S539 & .386 & & .386 & .386 & & & .386 & .386 & .386 \\
\hline D13S317 & .376 & & .376 & & .376 & .376 & & & .376 \\
\hline TH01 & .376 & .376 & .376 & .376 & .376 & & .376 & .376 & .376 \\
\hline D3S1358 & & .374 & .374 & .374 & .374 & .374 & .374 & 374 & .374 \\
\hline VWA & .362 & .362 & .362 & & .362 & .362 & .362 & .362 & .362 \\
\hline D8S1179 & & .358 & .358 & & & .358 & .358 & .358 & .358 \\
\hline D7S820 & .356 & & .356 & .356 & .356 & .356 & & & .356 \\
\hline D21S11 & & .336 & .336 & & & .336 & .336 & .336 & .336 \\
\hline FGA & & .328 & .328 & & .328 & .328 & .328 & .328 & .328 \\
\hline D18S51 & & .318 & .318 & & & .318 & .318 & .318 & .318 \\
\hline D19S433 & & & & & & & .390 & .390 & .390 \\
\hline Penta D & & & .346 & & & & & & \\
\hline D2S1338 & & & & & & & .315 & .315 & .315 \\
\hline Penta E & & .307 & .307 & & & & & & \\
\hline SE33 & & & & & & & & .283 & \\
\hline Number of STRs & 8 & 9 & 15 & 6 & 9 & 9 & 10 & 11 & 15 \\
\hline Overall $M(\mathrm{DZ})_{\text {approx }}$ & $5.93 \mathrm{E}-04$ & $9.47 \mathrm{E}-05$ & $2.96 \mathrm{E}-07$ & $3.86 \mathrm{E}-03$ & $1.89 \mathrm{E}-04$ & $9.60 \mathrm{E}-05$ & $3.03 \mathrm{E}-05$ & $8.57 \mathrm{E}-06$ & $3.42 \mathrm{E}-07$ \\
\hline $0 \mathrm{dds}_{\mathrm{Mz}: \mathrm{OZ}}$ & 1685 & 10,555 & $3,378,274$ & 259 & 5302 & 10,422 & 33,013 & 116,653 & $2,922,758$ \\
\hline MZ Certainty ${ }_{\text {avg }}(\%)$ & 99.94066 & 99.99053 & 99.99997 & 99.61360 & 99.98114 & 99.99041 & 99.99697 & 99.99914 & 99.99997 \\
\hline
\end{tabular}

Approximate Probability of a Caucasian DZ Twin Pair Sharing the Same Two Alleles

Note: Utilizing approximate $M(D Z)$ values reported in Table 4 (FULL SIB, $z_{2}$ column) of Presciuttini et al. (2002). 
to obtain probability of correct zygosity assignment for any combination of the 18 loci commonly used in the forensic practice. Table 4 contains $M(\mathrm{DZ})_{\text {approx }}$ and resulting probability of correct zygosity assignment for the commonly utilized multiplex STR kits listed in Table 1 . Moreover, because these markers are routinely used it should not be too difficult for researchers to obtain population-specific heterozygosity values appropriate for the twins under investigation.

Although the average certainty of correctly designating a twin pair sharing all alleles as MZ, is greater than $99 \%$ for all nine multiplex STR kits listed in Table 4, the chance of a DZ pair sharing all markers ranges from 1 in 259 (COfiler) to 1 in 3,378,274 (PPlex16). Indeed, as a result of determining accurate probabilities for the number of alleles being shared, researchers should keep in mind the possibility of genotyping errors and/or spontaneous mutations when determining zygosity. For example, the average probability of a DZ pair being $z_{2}$ at 8 of the 9 Profiler Plus loci range from 1.314E-04 to $2.058 \mathrm{E}-04$ (odds of 1 in 7609 to 1 in 4859), the odds of being $z_{2}$ at 7 loci range from 1 in 4585 to 1 in 2372, the odds of being $z_{2}$ at 6 loci range from 1 in 2739 to 1 in 1200, while the odds of a $\mathrm{DZ}$ pair being $z_{2}$ at 5 loci range from 1 in 1554 to 1 in 663 . However, error (including mutation) rates ranging from $0.25 \%$ to $2.38 \%$ (odds of 1 in 400 to 1 in 42) may be quite realistic (Ewen et al., 2000). Hence, it may be more likely that twin pairs sharing both alleles at 7 or 8 of the 9 Profiler Plus loci are $\mathrm{MZ}$ with genotyping errors than $\mathrm{DZ}$.

To this end, one can either calculate the overall average probability for the observed number of loci for which the pair of individuals are $z_{2}$ (and not $z_{2}$ ) and compare this to an assumed error rate(s), or use a fully parametric approach such as that implemented in the ECLIPSE2 program (Sieberts et al., 2002) which can provide the exact probability for a pair of individuals sharing particular alleles (i.e., the chance of a pair sharing common alleles is higher than for sharing rare alleles) and also take into account any correlation in allele sharing due to the use of linked markers. Given that a wide range of researchers may be interested in the latter approach, the author has implemented a userfriendly www interface ('ZygProb') to ECLIPSE2, allowing users to simply upload three input files to obtain ECLIPSE2 likelihood results for a pair of individuals sharing the uploaded marker alleles. Likelihoods are given, assuming user-specified error rates, for being a DZ pair (/full-sibling pair), halfsibling, unrelated, and $M Z$ pair. Additionally, the MZ/DZ likelihood ratio is given, which represents the odds in favour of the two individuals being an $\mathrm{MZ}$ pair compared to a DZ pair (i.e., odds greater than 1 indicate the pair is more likely to be MZ). Excel worksheets may also be downloaded from the ZygProb homepage (http://genepi.qimr.edu.au/general/daleN/ZygProb/) enabling easy calculation of exact and approximate random match probabilities $M(D Z)$ from allele frequency and heterozygosity data, respectively.

\section{Acknowledgments}

I thank Nick Martin and Peter Visscher for helpful suggestions. DRN was supported by an NHMRC RD Wright Fellowship \#339462.

\section{$\overline{\text { References }}$}

Bagdonavicius, A., Turbett, G. R., Buckleton, J. S., \& Walsh, S. J. (2002). Western Australian sub-population data for the thirteen AMPFISTR Profiler Plus and COfiler STR loci. Journal of Forensic Sciences, 47, 1149-1153.

Derom, R., Bryan, E., Derom, C., Keith, L., \& Vlietinck, R. (2001). Twins, chorionicity and zygosity. Twin Research, 4, 134-136.

Ewen, K. R., Bahlo, M., Treloar, S. A., Levinson, D. F., Mowry, B., Barlow, J. W., \& Foote, S. J. (2000). Identification and analysis of error types in highthroughput genotyping. American Journal of Human Genetics, 67, 727-736.

Heath, A. C., Nyholt, D. R., Neuman, R., Madden, P. A., Bucholz, K. K., Todd, R. D., Nelson, E. C., Montgomery, G. W., \& Martin, N. G. (2003). Zygosity diagnosis in the absence of genotypic data: An approach using latent class analysis. Twin Research, 6, 22-26.

Li, C. C. (1996). Population genetics of coincidental DNA matches. Human Biology, 68, 167-184.

Magnus, P., Berg, K., \& Nance, W. E. (1983). Predicting zygosity in Norwegian twins born 1915-1960. Clinical Genetics, 24, 103-112.

Presciuttini, S., Toni, C., Tempestini, E., Verdiani, S., Casarino, L., Spinetti, I., De Stefano, F., Domenici, R., \& Bailey-Wilson, J. E. (2002). Inferring relationships between pairs of individuals from locus heterozygosities. BMC Genetics, 3, 23-33.

Sieberts, S. K., Wijsman, E. M., \& Thompson, E. A. (2002). Relationship inference from trios of individuals, in the presence of typing error. American Journal of Human Genetics, 70, 170-180. 